\title{
MEDICAMENTOS ANTIDEPRESSIVOS: INSERÇÃO NA PRÁTICA BIOMÉDICA (1941 A 2006) A PARTIR DA DIVULGAÇÃO EM UM LIVRO-TEXTO DE FARMACOLOGIA
}

Silvia Cardoso Bittencourt

Sandra Caponi

Sônia Maluf

\section{Introdução}

Os termos psicoativo ${ }^{1}$ e psicotrópico ${ }^{2}$ denominam uma ampla categoria de drogas que agem no Sistema Nervoso Central (SNC), introduzidas na prática biomédica por volta da década de 1950, quando começa a "Era da Psicofarmacologia"3 (Healy 1998; Dagognet \& Pignarre 2005; Mazana, Pereira \& Cabrera 2002; Lopez-Munoz et alli 2004; Pignarre 2001). "Antidepressivo" refere-se a uma subcategoria dessas substâncias. Embora Henri Laborit, na década de 1940, tenha percebido o efeito tranquilizante de substâncias com ação anti-histamínica, a utilização de drogas para tratar pacientes com distúrbios mentais começa por volta de 1952, em um hospital psiquiátrico francês, com Pierre Denicker e Jean Delay. O conceito de neuroléptico, ${ }^{4}$ desenvolvido por Denicker e Delay (Healy 1998), provocou uma revolução terapêutica, dando força à psiquiatria biológica (Aguiar 2004; Mazana, Pereira \& Cabrera 2002; Dagognet \& Pignarre 2005).

Quando se ampliou o uso de fármacos industrializados, logo após a Segunda Guerra, observou-se que algumas drogas em teste para outros problemas de saúde melhoravam o humor de pacientes com sintomas depressivos, daí o termo antidepressivo. A primeira classe desses medicamentos foi a dos Inibidores da MAO: ${ }^{5}$ ao utilizar a iproniazida ${ }^{6}$ para tratar a tuberculose, percebeu-se que o humor dos pacientes melhorava. Porém, por possuir muitos efeitos colaterais, esta substância deixou de ser utilizada nas décadas seguintes (Dagognet \& Pignarre 2005).

Ao mesmo tempo, a investigação de substâncias relacionadas às fenotiazinas ${ }^{7}$ estava sendo explorada pelas maiores indústrias de medicamentos da época em função da descoberta de sua relação com a histamina, 
substância endógena reconhecida há anos, a qual passa a ser relacionada a várias situações clínicas (Dagognet \& Pignarre 2005; Fangmann et alli 2008; Healy 1997). A primeira fenotiazina foi sintetizada em 1883, no laboratório BASF (Badische Anilin und Sodafabriken), a partir do corante azul de metileno.

Ainda nessa década foi sintetizado o composto fenotiazínico iminodibenzil, inicialmente previsto para ser utilizado na indústria de roupas e "esquecido" nos "porões" da Geigy. ${ }^{8}$ Cerca de 50 anos depois, o diretor do setor farmacológico dessa indústria incentivou a pesquisa com os compostos fenotiazínicos na busca de medicamentos sedativos, e o iminodibenzil foi "ressuscitado" dos porões, dando origem a 42 derivados. Dentre estes, um deles deu origem à imipramina, que se estabeleceu como medicamento para tratar sintomas depressivos. Em 1957, os resultados dos testes com esta droga foram apresentados em um congresso de psiquiatria em Zurique, e ela passou a ser comercializada sob o nome de "Tofranil@". Nascia aqui a classe dos antidepressivos tricíclicos (Healy 1997; Fangmann et alli 2008).

No decorrer dos anos, novas classes de drogas, categorizadas a partir dos tipos de receptores com os quais interagem no organismo, foram incluídas no grupo dos antidepressivos. Na década de 1980, o desenvolvimento dos Inibidores Seletivos da Recaptação de Serotonina (ISRS) merece destaque, tanto pela difusão do seu uso por médicos não psiquiatras quanto pela ampliação do uso para outros diagnósticos além dos quadros caracterizados por sintomas depressivos (Horwitz \& Wakefield 2007; Turnquist 2002; Dagognet \& Pignarre 2005; Pignarre 2001). Por fim, nas últimas décadas do século XX e início do século XXI, outros medicamentos, como os inibidores seletivos da noradrenalina, foram lançados no mercado (Baldessarini 2006).

O objetivo deste estudo é analisar como esses medicamentos foram apresentados e como seu uso foi justificado para estudantes e profissionais médicos a partir do olhar da farmacologia, área do conhecimento que lida com o desenvolvimento e a validação de medicamentos. Se atualmente as informações técnico-científicas sobre essa ciência são encontradas em congressos médicos, artigos científicos e visitas de representantes de laboratório, entre outras fontes, algumas décadas atrás, a principal referência baseada no conhecimento científico disponível era o livro-texto. Leite (2004) lembra que o livro didático é um dos recursos utilizados para a atualização científica dos profissionais já formados, além de participar diretamente da formação dos estudantes.

Foram analisadas onze edições (de 1941 a 2006) do livro-texto de farmacologia Goodman and Gilman's: the pharmacological basis of therapeutics, amplamente utilizado em todo o mundo. Esta análise é feita por meio de 
uma abordagem histórico-etnográfica com auxílio da Análise de Discurso (AD). Vale lembrar que a perspectiva histórica permite que os acontecimentos sejam reunidos de um modo significativo, explicando, ainda que indiretamente, a realidade atual (Mommsen 1982:236-237). No caso deste estudo, optou-se por uma "micro-história" (Burke 2002:61) dos antidepressivos a partir do livro-texto, o que permitiu uma abordagem etnográfica desse livro, analisando como os antidepressivos foram apresentados em suas sucessivas edições, as categorias e os dispositivos retóricos utilizados, e mesmo as mudanças quanto aos sentidos enunciados em relação a estes. Burke (2002) aponta que, apesar do surgimento de críticas a estudos deste tipo, existem justificativas para seu emprego. Uma delas seria que:

A escolha de um exemplo individual a ser estudado em profundidade pode ser motivada pelo fato de representar a miniatura de uma situação que o historiador ou antropólogo já sabe (por outros motivos e com base em outras fontes) que é predominante (Burke 2002:64).

A análise histórica e etnográfica dessa situação singular permite, no caso deste estudo, olhar de outro modo um problema localizado no presente, que é o reducionismo biológico no uso de medicamentos, problema para o qual nos alertam diversos estudos de sociologia, antropologia e epistemologia. Isto porque o medicamento moderno, esse recurso tecnológico que é utilizado como estratégia terapêutica, faz parte da configuração ocidental moderna e ganha características que só podem ser validadas em função do contexto sociocultural em que está inserido, a partir de valores simbólicos e econômicos que traz consigo.

Não se pretende negar a existência de fatores biológicos na ação do medicamento, mas, concordando com Pignarre, entre a molécula, que possui uma ação biológica, e o medicamento, que é uma mercadoria administrada socialmente, há uma construção social que acaba por fazer deste último "uma maneira original de ligar o biológico ao social. Privilegiar um ou outro [desses aspectos] é atribuir-lhe [ao medicamento] um excesso de honra ou indignidade" (Pignarre 1999:15).

Assim, as obras foram analisadas, em um primeiro momento, como um todo: os prefácios, os índices, seções ou capítulos que tratavam da farmacologia como ciência e seções e capítulos que tratavam de substâncias psicoativas. Em um segundo momento, os capítulos sobre antidepressivos foram analisados de forma minuciosa. Com a $\mathrm{AD}$, pretendeu-se extrair as "não transparências" da linguagem (Orlandi 1999; Nogueira 2001, 2008) e aspectos como as "estratégias discursivas" (Van Dijk 1999:31), que incluem, 
por exemplo, questões relacionadas ao contexto (como a questão subjetiva da credibilidade de quem escreve o texto) e à estruturação do texto (por exemplo, a forma como os temas são dispostos ou a importância que se dá a cada um deles), assim como os conceitos e as categorias utilizados nas diferentes edições.

\section{Psicofarmacologia e medicamentos psicoativos no livro-texto}

\section{A época "pré-psicofarmacologia" (as edições de 1941 e 1955)}

Nas duas primeiras edições do livro-texto (1941 e 1955), não há referência a drogas "antidepressivas", nem se utiliza o termo medicamento "antidepressivo". Existem as seções "Drogas depressoras do SNC" e "Drogas estimulantes do SNC", indicando o tipo de ação predominante, com destaque para as primeiras. Sobre as segundas, os autores ressaltam que a ação estimulante não é seletiva no SNC, ela ocorre em todo o organismo (Goodman \& Gilman 1947 [1941]:254). Sobre as primeiras, dizem os autores:

Por conveniência, os depressores do sistema nervoso central podem ser divididos arbitrariamente em várias categorias. Uma classificação simples e prática é a seguinte: (a) anestésicos gerais [...]; (b) drogas sedativo-hipnótico-soporíferas [...]; (c) narcóticos [...]; e (d) analgésicos e antipiréticos ${ }^{9}$ [...] (Goodman \& Gilman 1947 [1941]:27).

Como destacam os autores, a classificação em subtipos é didática e arbitrária e, neste caso, o critério principal para fazer essa classificação foi clínico, baseado nos usos terapêuticos da prática médica cotidiana, embora destaquem que as drogas classificadas em uma categoria também podem ter outros efeitos. Citam o exemplo: "qualquer um dos sedativos pode ser administrado em doses suficientes para causar anestesia. [...] Por outro lado, os anestésicos gerais produzem analgesia (Goodman \& Gilman 1947 [1941]:27).

À medida que os autores discorrem sobre determinada droga, vão sendo elencados seus vários usos (fora o principal, que gerou a classificação) e possíveis formas de atuação farmacológica. Além das indicações clínicas principais (tais como anestésicos, sedativos), há referência de algumas delas para o tratamento de "distúrbios psiquiátricos". Por exemplo, ao falar sobre os "barbitúricos", medicamentos bastante utilizados naquela época, Goodman e Gilman destacam que: 
Em estados agudos de mania e, particularmente, no delirium tremens, os barbitúricos são valiosos [...]. O uso prolongado de barbitúricos para sedação de pacientes com desordens psiquiátricas pode resultar em confusão e acentuação dos sintomas e deve ser evitado (Goodman \& Gilman 1947 [1941]:147, grifos nossos).

O termo "depressão" ou "sintoma depressivo", no sentido de condição clínica, quase não aparece nessa edição. Foi identificado em duas ocasiões, referindo-se aos usos dos brometos: ${ }^{10}$

Pacientes que sofrem de depressão, arterioscleroses e febre estão particularmente sujeitos a desenvolver uma psicose por brometos em doses da droga que não parecem excessivas. [...] Obviamente nenhum paciente com um distúrbio mental ou nervoso não diagnosticado deve receber o halogênio ${ }^{11}$ até que seja bem estabelecido, sem dúvida, que a sua doença não é devida a uma ingestão prévia de brometo (Goodman \& Gilman 1947 [1941]:160).

O brometo não tem um uso racional no manejo de pacientes com depressão.

[...] Não são analgésicos, e seu uso para apaziguar a dor pode causar delírio (Goodman \& Gilman 1947 [1941]:165, grifos nossos).

Os argumentos em relação à classificação das drogas como "depressoras" e "estimulantes" do SNC continuam os mesmos na $2^{a}$ edição (1955), com a inclusão de novas categorias terapêuticas na primeira subclassificação. Além dos "anestésicos gerais", das "drogas sedativo-hipnótico-soporíferas", dos "narcóticos" e dos "analgésicos", há a inclusão dos "relaxantes musculares de ação central" e dos "antiepiléticos".

Embora os estudos com drogas para tratamento de pacientes psiquiátricos principiem na década de 1940, ainda não há referência sobre elas nessa $2^{\mathrm{a}}$ edição do livro (Goodman \& Gilman 1964 [1955]). No entanto, termos relacionados à "psiquiatria" aparecem de forma mais frequente na $2^{\mathrm{a}}$ edição do que na anterior, refletindo que, embora ainda não exista uma categoria de medicamentos psicoativos, este enfoque já está circulando na linguagem técnica da medicina da época. Por exemplo, na parte que trata dos barbitúricos (drogas às quais são dedicadas várias páginas nessas duas edições), há um subtítulo denominado "Usos neuropsiquiátricos" na edição de 1955, em que os autores explicam o uso de tais substâncias nesse âmbito:

[Além do tratamento de outros sintomas,] os barbitúricos são utilizados com propósito de investigação em neuropsiquiatria e para o diagnóstico e tratamento de várias desordens mentais. Os termos narcoanálise e narcoterapia foram 
cunhados para designar esses usos especializados. [...] O objetivo é estabelecer um estado de relaxamento e sedação profunda, no qual as inibições são removidas e o paciente pode ser induzido a comunicar livremente o material de conflito emocional que está causando seus sintomas (narcocatarse) (Goodman \& Gilman 1964 [1955]:141; grifos no original).

Os autores complementam que a "narcocatarse" serve para fazer o diagnóstico diferencial entre quadros neuróticos (como a histeria de conversão e fadiga de combate ${ }^{12}$ ) e quadros psicóticos, e para o tratamento dos primeiros. O conhecimento farmacológico neste caso serve apenas para dizer quais doses provocam o efeito sedativo (Goodman \& Gilman 1964 [1955]:141) para que o tratamento clínico seja instituído.

\section{A psicofarmacologia ganha espaço: medicamentos para problemas psiquiátricos no livro-texto (edições de 1965 a 1990)}

A abordagem dos problemas psiquiátricos e dos medicamentos antidepressivos como temas de capítulos ou seções específicas no livro de farmacologia acontece na $3^{a}$ edição (Goodman \& Gilman 1965). Esta sofre uma reorganização de temas, com a substituição das seções "Drogas depressoras do SNC" e "Drogas estimulantes do SNC" pela seção "Drogas que atuam no SNC". Além dos 17 capítulos que faziam parte das duas edições anteriores, a $3^{a}$ edição ganha um novo, o das "Drogas utilizadas na terapia de desordens psiquiátricas", que começa considerando o papel desses medicamentos:

Embora o interesse nos efeitos psicológicos dos agentes farmacológicos seja tão antigo quanto a humanidade, os usos de drogas para o tratamento das desordens psiquiátricas tornou-se disseminado somente a partir da metade dos anos de 1950. Medicamentos têm encontrado seu papel nas práticas psiquiátricas de orientação mais analítica, e na prática da medicina eles são usados em grande escala para modificar atitudes e emoções dos pacientes. Nesta última direção, eles são frequentemente abusados. Ninguém vai negar que o indivíduo claramente psicótico ou severamente deprimido está em situação urgente de terapia, e as drogas agora desempenham um papel maior no tratamento dessas desordens severas. No entanto, o vasto número de indivíduos neuróticos poderia ser auxiliado mais por médicos simpáticos e compreensivos do que por drogas, se o médico puder despender mais tempo ouvindo esses pacientes e discutindo seus problemas com eles (Jarvik ${ }^{13}$ 1965:159, grifos nossos). 
$\mathrm{Na} 4^{\mathrm{a}}$ edição (Goodman \& Gilman 1970), que continua de forma geral muito semelhante à $3^{\mathrm{a}}$, este trecho se mantém. Os medicamentos utilizados na área de psiquiatria são trazidos como recurso terapêutico, e os profissionais médicos devem discernir quando há necessidade de aliviar o sofrimento, evitando seu uso desmedido, como aparece no excerto acima, apenas para "modificar atitudes e emoções". Há o alerta para que não ocorra o uso abusivo dessas drogas, ao mesmo tempo em que se sinaliza que já existe uso indevido.

$\mathrm{Na} 3^{\mathrm{a}}$ edição, são apontados aspectos psicológicos, sociais e econômicos relacionados aos distúrbios psiquiátricos e feitas considerações sobre o papel dos medicamentos. São abordados os limites e as vantagens do tratamento farmacológico, sempre contextualizando o assunto. O autor escreve nesta edição um trecho que se mantém idêntico na seguinte (1970):

É importante assinalar que um diagnóstico psiquiátrico, por si só, não determina a natureza da terapia medicamentosa. [...]. É necessário um julgamento clínico considerável na determinação de quais drogas são necessárias para determinado paciente em uma situação particular. [...]. A única forma de tratamento que um psiquiatra sozinho pode administrar simultaneamente a uma série de centenas de pacientes é o tratamento medicamentoso. No entanto, a farmacoterapia tem limitações claras no tratamento dos distúrbios psicológicos. As drogas não podem modificar diretamente influências hereditárias, relacionadas a estruturas familiares ou situações socioeconômicas de um indivíduo. Elas podem modificar a reação do paciente ao seu meio ambiente e seu funcionamento emocional e intelectual, mas o comportamento em curso vai continuar sendo determinado em grande parte pelas repostas aos estímulos que permanecem (Jarvik 1965:161; 1970:153, grifos nossos).

Esta última frase reforça o uso dos medicamentos como mais um recurso terapêutico para lidar com os sintomas. Há clareza de que o paciente é o foco, e o contexto no qual está inserido é valorizado. É possível perceber que a abordagem é semelhante àquela descrita nos séculos precedentes, na qual se leva em conta o contexto individual e social daquele que tem determinados sintomas (Horwitz \& Wakefield 2007). Mesmo que a argumentação para o uso das drogas psicoativas esteja relacionada a questões práticas, como a possibilidade de atender a muitos pacientes, há destaque para que se leve em conta o contexto maior daquele que vai ser tratado (Jarvik 1965, 1970), caracterizando uma abordagem não reducionista.

Na edição seguinte (Goodman \& Gilman 1975), o capítulo que trata das drogas para doenças psiquiátricas mantém o mesmo nome, mas passa a ser 
escrito por outro autor e tem sua introdução modificada. São apresentadas questões como o uso ampliado de medicamentos psicoativos:

Vinte por cento das prescrições escritas em uma comunidade média nos Estados Unidos são para medicações que se destinam a afetar os processos mentais, nomeadamente estimular, sedar ou modificar de outra forma o comportamento (Byck $^{14}$ 1975:152).

Há ainda referência às dificuldades em como estabelecer os diagnósticos psiquiátricos para o uso das drogas que possuem determinada indicação clínica:

O uso de drogas para o tratamento das desordens psiquiátricas é complicado por muitas das incertezas e imprecisões diagnósticas que têm importunado a psiquiatria. [...] Em psiquiatria, apesar dos melhores esforços, a confusão das entidades diagnósticas é antes uma regra do que uma exceção e, consequentemente, o uso de drogas psicotrópicas frequentemente sofre a ausência da precisão e da elegância possíveis em outras áreas da medicina (Byck 1975:152).

O autor complementa que a falta de diagnóstico preciso não deve impossibilitar o uso das drogas que sejam benéficas ao paciente e lembra que subsídios para estabelecer a terminologia diagnóstica podem ser encontrados "no Manual de diagnóstico e estatística de saúde mental II"15 (1968), da Associação Americana de Psiquiatria" (Byck 1975:153, grifo no original). Destaca também que as drogas psicoativas, ao longo de sua história, têm sido utilizadas em duas vias diferentes, enumeradas na seguinte ordem: "a primeira foi o uso de drogas para modificar comportamentos normais e para produzir modelos de loucura [madness]. A segunda foi para aliviar ou curar doenças mentais" (Byck 1975:153).

Sobre aspectos históricos, o autor lembra que a pesquisa psicofarmacológica teve uma rápida expansão na década de 1960 e, em consequência, houve aumento do uso dos medicamentos psicoativos. Ele completa: "além disso, muita atenção tem sido dada à responsabilidade no tratamento com drogas psicoterapêuticas, e uma visão equilibrada de suas vantagens e desvantagens está começando a emergir" (Byck 1975:153). Esta última frase traz novamente a ideia de usos "não equilibrados" ou abusivos. Mas a questão do mau uso não é explicitada nesta $5^{\mathrm{a}}$ edição como havia sido feito nas $3^{\mathrm{a}}$ (1964) e $4^{\mathrm{a}}$ (1970) edições.

O capítulo que trata das drogas para doenças psiquiátricas na $6^{\mathrm{a}}$ edição (Gilman, Goodman \& Gilman 1983 [1980]) começa com comentários sobre 
as drogas prescritas nos Estados Unidos. Segundo o autor do capítulo, Baldessarini ${ }_{1}^{16}$ cerca de $20 \%$ seriam aquelas que atuam em processos mentais (1983:346), tal como referido na edição anterior. São consideradas, neste capítulo do livro que trata das drogas para uso psiquiátrico, três categorias de drogas: a) os antipsicóticos, também chamados neurolépticos, utilizados para as psicoses; b) os chamados estabilizantes do humor (o exemplo citado é o dos sais de lítio) e antidepressivos, que também são denominados pelo autor de "agentes elevadores do humor"; e c) os agentes ansiolítico-sedativos, "particularmente os benzodiazepínicos (BZD)", que são empregados nos estados de ansiedade (:346). Novamente, como na $5^{\mathrm{a}}$ edição, são citadas as incertezas do diagnóstico psiquiátrico, e nesta $6^{\mathrm{a}}$ edição sugere-se que os leitores se dirijam ao Manual de diagnóstico e estatística de saúde mental (DSM) da Associação Americana de Psiquiatria (:347), demonstrando o vínculo que a farmacologia estabelece com os critérios clínicos vigentes em cada época.

Há ainda a referência às pesquisas com os medicamentos psicotrópicos e suas relações com "possíveis bases biológicas para as doenças psiquiátricas", que o autor diz serem auxiliadas por "uma disciplina médica conhecida como psiquiatria biológica". Ele complementa: "Embora às vezes haja desacordo entre psiquiatras a respeito do diagnóstico e das indicações para vários tratamentos, essas incertezas não invalidam os muitos efeitos salutares de medicamentos sobre os sintomas mentais" (:347).

Essas afirmações nos remetem à questão do conhecimento científico e seus dois âmbitos de desenvolvimento: um relacionado à busca de explicações causais e o outro, ao desenvolvimento de produtos técnicos, como destacam Lacey (1998) e Lewontin (1991). Este último, ao referir-se às duas funções da ciência - fornecer meios para a manipulação de recursos da natureza proporcionando mudanças no modo de vida, e buscar explicações para "como as coisas são e como funcionam" (Lewontin 1991:4) - lembra que muitas vezes elas estão completamente separadas, embora possam também estar relacionadas. No caso dos medicamentos psicoativos, se há a busca das explicações causais, este aspecto está em desenvolvimento e tem oferecido apenas respostas parciais. Embora se afirme que "a introdução de hipóteses farmacologicamente orientadas referentes às bases biológicas de doenças mentais graves encorajou a pesquisa racional em psiquiatria" (Baldessarini 1983:348), não são estabelecidas relações causais a respeito das doenças mentais a partir da farmacologia até a $6^{a}$ edição do livro-texto. O que existe são hipóteses, e o uso de medicamentos baseia-se nos sucessos da clínica: mesmo que não se tenha um diagnóstico preciso, a utilização de medicamentos pode aliviar sintomas. 
A $7^{\text {a }}$ (Gilman et alli 1985) e a $8^{\text {a }}$ (Gilman et alli 1990) edições incluem o capítulo que trata das drogas para doenças psiquiátricas escrito por Baldessarini, o mesmo autor da $6^{a}$ edição (1980), e o texto se mantém praticamente idêntico. Sobre o diagnóstico diferencial das doenças psiquiátricas para o uso das drogas que são indicadas em diferentes situações clínicas, não há mais a referência explícita aos DSM, mas uma referência indireta, através de citações bibliográficas no decorrer do texto (Baldessarini 1990:384).

Tanto a $7^{\mathrm{a}}$ como a $8^{\mathrm{a}}$ edição do Goodman \& Gilman têm na introdução a seguinte consideração: "alguns aspectos principais da nosologia psiquiátrica são sumarizados brevemente aqui, e informações adicionais são fornecidas nas discussões das classes de drogas específicas" (Baldessarini 1985:388; 1990:384). Sobre essa nosologia são apresentadas classificações clínicas, como a diferenciação entre: a) psicoses; b) neuroses; e c) desordens afetivas maiores ou desordens maníaco-depressivas, primariamente causadas por alterações das emoções e do humor, e que podem ser classificadas como "doença bipolar", "doença unipolar" ou "episódios moderados de depressão".

Sobre esta última categoria, o autor refere que: "em adição [à doença bipolar e unipolar], a depressão pode ocorrer como uma desordem moderada ou como um sintoma de outras doenças psiquiátricas ou médicas" (Baldessarini 1990:384). Segundo o autor, os diagnósticos incluídos nessas três categorias de classificação têm sintomas que podem se sobrepor (Baldessarini 1990:384), corroborando a colocação dos autores das edições anteriores sobre a dificuldade do diagnóstico em psiquiatria.

É possível observar, a partir da leitura dessas edições do livro-texto, que tanto a inclusão em alguma categoria quanto os tratamentos dependem do diagnóstico clínico realizado. Este, por sua vez, sofre a influência da percepção subjetiva do médico e do que é considerado como doença em determinado momento histórico.

Mesmo com a busca de uma relação entre as doenças ligadas ao comportamento e determinadas substâncias endógenas, também nas $7^{\mathrm{a}}$ e $8^{\mathrm{a}}$ edições do livro-texto não é a partir da farmacologia que ocorre a classificação das doenças psiquiátricas, mas sim de critérios da clínica. Cabe destacar que na $1^{a}$ edição do livro os autores afirmam que "embora a farmacologia seja, em si mesma, uma ciência médica básica, ela recebe contribuições de muitas disciplinas médicas e contribui livremente com vários assuntos e técnicas dessas disciplinas, quer sejam estas clínicas ou pré-clínicas" (Goodman \& Gilman 1947 [1941]:v). 


\section{Final do século XX e início do século XXI: os antidepressivos ganham destaque entre os psicofármacos (as edições de 1996, 2001 e 2006)}

A $9^{a}$ edição (Hardman et alli 1996) traz reformulações na organização do livro como um todo. Por cinco edições (da $3^{\mathrm{a}}$ à $8^{\mathrm{a}}$ ), o capítulo "Drogas no tratamento das desordens psiquiátricas" incluiu três categorias de medicamentos: a) drogas para psicoses; b) drogas para ansiedade; c) drogas para depressão ( $3^{\mathrm{a}}$ edição) ou drogas para transtornos de humor $\left(4^{\mathrm{a}}, 5^{\mathrm{a}}, 6^{\mathrm{a}}\right.$, $7^{\mathrm{a}}, 8^{\mathrm{a}}$ edições). A partir da $9^{\mathrm{a}}$ edição, este capítulo é desmembrado em dois: "Drogas e o tratamento das desordens psiquiátricas: psicose e ansiedade" (Cap.18) e "Drogas e o tratamento das desordens psiquiátricas: depressão e mania" (Cap.19).

Nas $10^{\mathrm{a}}$ (Hardman, Limbird \& Gilman 2001) e $11^{\mathrm{a}}$ (Brunton, Lazo \& Parker 2006) edições, essas drogas são descritas ainda em dois capítulos, mas com seus títulos reformulados. Na $10^{\mathrm{a}}$ edição, eles têm os títulos “Drogas e o tratamento das desordens psiquiátricas - depressão e desordens de ansiedade" e "Drogas e o tratamento das desordens psiquiátricas - psicose e mania". Já na $11^{\mathrm{a}}$ edição são intitulados "Terapia medicamentosa da depressão e desordens de ansiedade" e "Farmacoterapia das psicoses e mania".

Essas mudanças nos "arranjos" das drogas por capítulos, relacionando-as com os diagnósticos e os sintomas em combinações diferentes, demonstram mais uma vez o quanto as classificações nessa área são flexíveis. Outro aspecto a ser destacado é a ausência de definições de conceitos nas últimas edições, em particular na $11^{\mathrm{a}}$ (Brunton, Lazo \& Parker 2006). Nesta, até mesmo o termo "farmacologia" não é conceituado ou definido, algo que aconteceu em todas as outras edições. Em relação às categorias de drogas, se nas edições precedentes havia a definição do que seriam drogas "depressoras" ou "estimulantes", "antipsicóticos" ou "neurolépticos", entre outras conceituações, na $11^{\mathrm{a}}$ edição os termos são utilizados sem que se explore seu significado.

Sobre esta questão, vale lembrar que são as opacidades do discurso, mais do que as transparências, que interessam em uma análise crítica sobre o que está sendo dito (Orlandi 1999, 2005). Ao não se colocar o que significam essas denominações, permite-se, por exemplo, a interpretação (naturalizada, como se só esta fosse possível) de que as drogas "antidepressivas" e "antipsicóticas" agem "contra" as depressões e as psicoses, quando esta não é a única interpretação possível, pois essas drogas podem estar apenas minimizando sintomas e não provocando a "cura", ou ainda, podem estar sendo usadas para tratar sintomas não relacionados a esses diagnósticos. 
Por exemplo, a categoria nosológica "ansiedade", que era abordada em um subtítulo (antes da $9^{a}$ edição), ganha um capítulo em que está associada às psicoses ( $9^{\mathrm{a}}$ edição) e depois à depressão ( $10^{\mathrm{a}}$ e $11^{\mathrm{a}}$ edições). Os ISRS passaram a ser indicados para situações relacionadas à "depressão", e também para os chamados distúrbios de ansiedade, associados ou não aos benzodiazepínicos, como no caso do diagnóstico de Transtorno de Ansiedade Generalizada (TAG) e do Transtorno de Ansiedade Social (TAS).

Conrad (2007) aborda o surgimento destas duas entidades nosológicas a partir do interesse de um laboratório farmacêutico em lançar no mercado um ISRS (paroxetina), quando a fluoxetina já detinha o espaço para tratar a depressão e havia a necessidade de encontrar outro nicho para a primeira. A "troca" dos BZD pelos ISRS (ou pelo menos a associação de ambos) pode ter suas origens na introdução do diagnóstico de "depressão" de forma ampliada, no meio médico, com a ajuda da própria indústria farmacêutica.

Sobre esta questão, Healy (1997) lembra que uma possibilidade de narrativa da descoberta dos medicamentos para tratar a depressão foi a sua "descoberta" pela indústria farmacêutica, no final dos anos 1950 (:74), com o desenvolvimento da amitriptilina, um composto quase idêntico à já conhecida imipramina. A amitriptilina foi pesquisada ao mesmo tempo (o que não costuma ser comum no âmbito da indústria farmacêutica) pelas companhias Merck, Roche e Lundbeck. Healy assinala que "quando se tornou claro que havia dinheiro a ser feito, com sucesso, com os antidepressivos", as duas companhias romperam com a Merck, mas esta saiu vencedora porque havia patenteado a droga especificamente para a depressão (:75).

Podem ser citados dois fatores para que a substituição do amplo uso dos BZD tenha ocorrido apenas com os ISRS no final do século XX: o primeiro relacionado ao espaço já ocupado pelos BZD nas décadas de 1960 e 1970, e o segundo relacionado aos efeitos colaterais que ainda são intensos com os tricíclicos (embora bem menos do que os dos Inibidores da MAO). Sobre estes fatores, Healy (1997) afirma:

Em essência, no entanto, a Merck não vendeu apenas amitriptilina, ela vendeu uma ideia. A amitriptilina tornou-se o primeiro dos antidepressivos a ser vendido em volumes substanciais. Os anos de 1960, no entanto, eram um mundo no qual o Librium ${ }^{\circledR}$ e o Valium ${ }^{\circledR}{ }^{17}$ eram reis, o que significa que, comparado com as percepções correntes da época, o tamanho do mercado da depressão não hospitalizada era provavelmente considerado relativamente pequeno. Não havia, nessas circunstâncias, nenhum espaço para uma diversidade de conceitos (para energizantes psíquicos, timolépticos ${ }^{18}$ e antidepresssivos), e tampouco foram reconhecidas clinicamente claras diferenças entre os diversos grupos de 
compostos conhecidos, eles foram colocados sob a ampla noção "guarda-chuva" de antidepressivos, suficiente para manter as vendas até que o eclipse dos BZD conduzisse a uma expansão do mercado de antidepressivos e à ampla descoberta desses medicamentos, que só aconteceu com os ISRS (Healy 1997:76).

Vale lembrar que se os barbitúricos foram drogas amplamente utilizadas na primeira metade do século XX (inclusive de forma abusiva, como é possível observar em passagens do livro-texto nas três primeiras edições), os compostos benzodiazepínicos (BZD) foram "as" drogas psicoativas das décadas de 1960 e 1970, mantendo seu uso nas décadas seguintes, apesar dos alertas de uso abusivo e da possibilidade de provocarem dependência nos usuários (Auchewski et alli 2004; Nastasy, Ribeiro \& Marques 2008; WHO 1983; Woods, Katz \& Winger 1988). Já os ISRS têm sido utilizados amplamente a partir da década de 1990, e esse uso também pode estar sendo abusivo, como apontam autores como Pignarre (2001) e Turnquist (2002).

\section{As diferentes classes de medicamentos antidepressivos}

\section{Inibidores da MAO: os primeiros antidepressivos}

Os primeiros medicamentos abordados em "Drogas para depressão" na $3^{a}$ edição do livro-texto são os Inibidores da MAO (Goodman \& Gilman 1965). Vale destacar que nesta edição não há referência ao termo "antidepressivos", nem no decorrer do texto, nem no índice alfabético remissivo. Este termo aparece pela primeira vez em subtítulo do item "Drogas para depressão" da $4^{\mathrm{a}}$ edição (1970), sem constar, no entanto, no índice remissivo como palavra de busca, refletindo sua introdução na linguagem da época, mas ainda não de forma disseminada. O índice alfabético da $6^{a}$ edição não pôde ser pesquisado, sendo na $7^{a}$ edição a primeira vez em que o termo "antidepressivo" foi observado.

Não há dúvidas de que os IMAO foram as primeiras drogas utilizadas em pacientes com sintomas depressivos (Dagognet \& Pignarre 2005; Healy 1997). Em todas as edições do livro-texto de Goodman e Gilman, sua introdução nas pesquisas pré-clínicas e clínicas é referida a partir do início da década de 1950, enquanto os tricíclicos só aparecem a partir da segunda metade dessa mesma década. Porém, Healy (1997) aponta que em diferentes momentos da história considerou-se uma ou outra classe e seus "descobridores" como pioneiros na utilização de drogas com o propósito de tratar pacientes depressivos. 
Uma das razões para esse reconhecimento "tardio" é que os IMAO foram pouco valorizados por seus efeitos colaterais facilmente reconhecíveis, e os tricíclicos foram considerados os medicamentos que "abriram" esse espaço na terapêutica. Healy (1997) lembra que a expansão de ambos como recurso terapêutico ocorreu na década de 1960, com o lançamento dos tricíclicos no mercado. Por outro lado, a possível relação da serotonina com a depressão foi apontada pelos pesquisadores que trabalharam com os IMAO como drogas antidepressivas já na década de 1950, tese que ganhou força nas últimas décadas do século XX e que reforça o papel dos IMAO como os primeiros antidepressivos (Healy 1997). Isto porque os IMAO atuam diminuindo a recaptação da enzima monoamina oxidase, que tem papel importante no metabolismo das chamadas aminas biogênicas (incluindo dopamina, noradrenalina, adrenalina e serotonina).

$\mathrm{Na} 3^{\mathrm{a}}$ (1965) e na $4^{\mathrm{a}}$ (1970) edições do livro-texto, a abordagem dos IMAO se inicia da seguinte forma:

Até o final da década de 1950, não havia tratamento farmacológico amplamente aceito para depressão. [...] A introdução dos inibidores da MAO e, mais tarde, dos derivados dibenzazepínicos ${ }^{19}$ gerou algum otimismo em relação às várias síndromes depressivas, já que estudos clínicos controlados têm indicado que ambos os grupos de agentes são de fato efetivos em certos casos. [...] No entanto, a relação entre a inibição da MAO e as ações terapêuticas dessas drogas não está estabelecida (Jarvik 1965:181; 1970:153, grifo nosso sublinhado e do original em itálico).

O otimismo que aparece com o resultado dos usos dessas drogas em pacientes com sintomas depressivos é precedido do adjetivo "algum", provavelmente em função dos divergentes resultados nos estudos clínicos. Completando:

A eficácia terapêutica dos inibidores da MAO não está bem confirmada. Alguns investigadores afirmam que suas experiências com os inibidores da MAO têm sido desapontadoras [...]; outros relatam, completamente entusiasmados, que essas drogas são efetivas [...] (Jarvik 1965:203; 1970:191).

Dependendo do observador, do paciente e da situação, a depressão pode ser vista como um sintoma, uma síndrome ou uma entidade nosológica, e a perda de uniformidade em classificar os diversos quadros clínicos considerados sob a rubrica de depressão constitui o maior obstáculo para a definição de qualquer modalidade de tratamento (Jarvik 1965:202; 1970:191, grifo nosso). 
Na $5^{a}$ edição (Byck 1975:186) também aparece a parte grifada da citação acima. No decorrer das próximas edições, é possível observar a tentativa de objetivação do diagnóstico da depressão, sempre a partir de critérios clínicos. Também há no texto comentários sobre as possíveis ações dessas drogas no organismo derivados de estudos farmacológicos, sem que a indicação terapêutica parta da farmacologia. Nas $6^{\mathrm{a}}, 7^{\mathrm{a}}$ e $8^{\mathrm{a}}$ há referência à limitação do uso dos IMAO (Baldessarini 1983:377; 1985:423; 1990:414), apontando seu papel secundário no tratamento da depressão em relação aos compostos tricíclicos. Nas $9^{\mathrm{a}}, 10^{\mathrm{a}}$ e $11^{\mathrm{a}}$ edições, eles são citados, mas têm menos destaque na obra, sempre em função de seus efeitos colaterais exacerbados.

\section{Os tricíclicos - ampliando o mercado de medicamentos para tratar sintomas depressivos}

A segunda classe de medicamentos apresentada na $3^{\text {a }}$ edição, os "compostos dibenzazepínicos", é descrita no segundo subtítulo do subitem "Drogas para depressão". Assim inicia sua apresentação na $3^{a}$ edição (1965), que tem a parte grifada repetida na $4^{\mathrm{a}}$ edição (1970):

Os derivados dibenzazepínicos, imipramina e amitriptilina são as drogas mais amplamente utilizadas para o tratamento da depressão. Eles podem ser considerados sucessores dos inibidores da $\mathrm{MAO}$, os quais por muitos anos foram considerados os únicos agentes efetivos disponíveis para essa condição. [...] Embora pouco se conheça sobre a etiologia da depressão endógena ou os mecanismos de ação das drogas dibenzazepínicas, sua eficácia em aliviar tal condição tem sido bem estabelecida (Jarvik 1965:198; 1970:186, grifo nosso).

Na $5^{\mathrm{a}}$ edição, o subitem "Drogas usadas no tratamento das desordens afetivas" traz no seu início dois parágrafos que falam sobre as desordens mania e depressão, consideradas "sintomas primários". Nesta parte, o autor destaca que esses sintomas, caracterizados por extremos de humor, podem ser acompanhados de psicoses ou, ao contrário, as psicoses podem apresentar esses sintomas no seu curso. Além disso, "mudanças severas de humor sem psicose podem ser acompanhadas de ansiedade" (Byck 1975:174). Após essas considerações, afirma que a escolha de um antipsicótico ou um antidepressivo fica a critério do médico em função da história do paciente e do quadro clínico.

A seguir, os tricíclicos são a primeira classe de medicamentos abordada, com a descrição das características farmacológicas dessas drogas. O autor 
afirma que "sua eficácia em aliviar a depressão tem sido bem estabelecida", sem referência ao que seria "depressão" (Byck 1975:174). No decorrer do texto sobre os tricíclicos, há referência à "fisiopatologia aminérgica da depressão na tentativa de explicar as ações das drogas antidepressivas" (Byck 1975:176). No entanto, nessa mesma edição há considerações sobre a incerteza quanto ao modo de atuação dessas drogas:

A ação dos antidepressivos tricíclicos no metabolismo das catecolaminas e indolaminas no cérebro tem contribuído significativamente para a "hipótese bioaminérgica" da depressão. O campo é tão complexo que somente um breve esboço é apresentado aqui. [...] Todos os antidepressivos tricíclicos bloqueiam a recaptação da noradrenalina pelos terminais adrenérgicos dos nervos. [...] alguns são mais potentes em bloquear a recaptação de $5-\mathrm{HT} \cdot{ }^{20}[\ldots]$ A relação desses efeitos com as ações dos antidepressivos tricíclicos na depressão humana não é conhecida (Byck 1975:176).

Na 6a edição (1980, 1983 brasileira), também no subitem que trata dos "medicamentos usados nos distúrbios de humor", antes de entrar no tema das drogas específicas, há uma pequena introdução. Nesta são abordados os quadros de "distúrbios afetivos" (que incluem os diagnósticos de mania e depressão), sua relação com os quadros de psicoses e as confusões que podem ocorrer no diagnóstico diferencial, influenciando a escolha dos medicamentos. A abordagem é muito semelhante à da $5^{\mathrm{a}}$ edição (1975).

De forma geral, essas quatro edições trazem tanto as dificuldades de diagnóstico diferencial entre as categorias de doenças psiquiátricas como o fato de mania e depressão poderem ser um sintoma ou uma entidade nosológica, porém não de forma tão explícita quanto nas $3^{\mathrm{a}}$ e $4^{\mathrm{a}}$ edições. Se na $5^{\mathrm{a}}$ edição a hipótese bioaminérgica foi tratada no decorrer do tema "tricíclicos", nas $6^{\mathrm{a}}, 7^{\mathrm{a}}$ e $8^{\mathrm{a}}$ edições ela faz parte dessa pequena introdução, antes das classes específicas de drogas, com a seguinte consideração: os IMAO e os ATD tricíclicos têm ação sobre as monoaminas biogênicas, e essa ação leva à especulação (termo usado na 8a edição de Goodman \& Gilman pelo autor Baldessarini 1990:405, grifo nosso) de uma "base biológica" relacionada com alterações nessas substâncias e os "distúrbios de humor".

Apesar disso, o autor afirma: "No entanto, há pouca evidência direta sobre essa visão, e não está claro o quanto as ações nos sistemas monoaminérgicos são cruciais para os efeitos clínicos da maioria das drogas antidepressivas" (Baldessarini 1985:413), ou desta forma, na $8^{a}$ edição: "No entanto, a evidência dessa visão é limitada e inconsistente" (Baldessarini 1990:405). 
Na 9a edição (1996), os tricíclicos passam a dividir a indicação, como medicamentos de primeira escolha, com os ISRS (Baldessarini 1996:432439), que eram considerados "atípicos" até a $8^{\mathrm{a}}$ edição. Nas $10^{\mathrm{a}}$ e $11^{\mathrm{a}}$ edições, os tricíclicos ainda aparecem, de forma semelhante à da $9^{a}$ edição, mas dividem o espaço, como drogas de primeira indicação, com os medicamentos incluídos na categoria dos ISRS.

Vale lembrar que se nas primeiras edições do livro-texto havia referência ao papel do médico no diagnóstico diferencial dos quadros clínicos e na sua relação com o paciente como fatores importantes na escolha do medicamento, nas últimas edições (em especial a partir da $10^{\mathrm{a}}$ ) este aspecto não é mais destacado, com citação apenas de referências sobre as classificações diagnósticas, como os DSM. O que se quer destacar é que esses aspectos, antes citados, deixam de aparecer nas edições mais recentes. Não citá-los ou negá-los demonstra a possibilidade de reducionismo explicativo.

\section{Os Inibidores Seletivos da Recaptação da Serotonina (ISRS)}

Os ISRS aparecem nas $7^{\mathrm{a}}$ e $8^{\mathrm{a}}$ edições na categoria "antidepressivos atípicos", com poucas linhas sobre sua atuação, apenas citando seu surgimento. Na 9a edição, deixam de pertencer a esta categoria. Assim os autores apresentam essas drogas na $9^{a}$ edição, que tem outros "atípicos" que não mais os ISRS:

Recentemente, após décadas de progresso limitado, uma série de antidepressivos inovadores tem emergido. A maior parte deles, como a "fluoxetina", são caracterizados como inibidores da inativação da recaptação ativa da serotonina [...]; outros [...] têm uma neurofarmacologia menos bem definida e podem ser considerados "atípicos". Embora a eficácia dos novos agentes não tenha demonstrado ser superior aos agentes mais antigos, sua segurança relativa e tolerabilidade têm levado à sua rápida aceitação como os antidepressivos mais comumente prescritos ${ }^{21}$ (Baldessarini 1996:432, grifo nosso).

O destaque é para a questão da não superioridade de eficácia em relação a compostos mais antigos. Diferentemente dos IMAO e dos tricíclicos, que têm a história de seu desenvolvimento citada nas diferentes edições do livro de Goodman e Gilman, não há referência ao desenvolvimento dos ISRS, nem aos seus aspectos históricos.

Sobre estes compostos, Healy lembra que se a adrenalina, a dopamina e a noradrenalina foram investigadas na América, a Europa se interessou mais 
pela serotonina. Em 1963, aparecem estudos com o triptofano (o aminoácido precursor da serotonina) e sua relação com os IMAO na Inglaterra. Nos anos seguintes, isso também acontece na Holanda, Suíça e Suécia. Foi neste último país, em Gotemburgo, que três pesquisadores iniciaram estudos com os antihistamínicos bromofeniramina e clorfeniramina (Healy 1997:165). Provocando uma pequena alteração na molécula da primeira substância, Hans Corrodi, que trabalhava em uma subsidiária da Astra, ${ }_{1}^{22}$ produziu a zimelidina em 1972, o primeiro inibidor específico da recaptação de serotonina. Esta droga, patenteada para uso como antidepressivo e liberada na Europa, foi retirada do mercado em 1982 por ter sido relacionada ao aparecimento de casos da Síndrome de Guillain Barré (Healy 1997:165; Lundbeck 2000:17). Nos Estados Unidos, o processo de aprovação pela FDA não foi concluído por causa deste efeito colateral, e a droga não chegou a ser comercializada.

Em outros locais, pesquisas com drogas inibindo a recaptação da serotonina foram desenvolvidas. Em 1983 foi lançado o citalopram na Dinamarca pela Lundbeck ${ }^{23}$ que, embora não tenha feito tanto sucesso na época quanto a fluoxetina (lançada alguns anos depois), continua no mercado ainda hoje. A fluoxetina, com estudos iniciados também na década de 1970, foi lançada pela Lilly ${ }^{24}$ nos Estados Unidos em $1987^{25}$ e, nos anos seguintes, em vários países da Europa. Outros compostos da mesma classe surgiram na década de 1990, como a sertralina e a paroxetina (Healy 1997:166-167).

A fluoxetina representou os ISRS por anos, como "estrela" dessa classe de medicamentos, caracterizando uma época em toda a medicina, e não apenas na psiquiatria. Em 1972, segundo Healy, quando os pesquisadores da Lilly estudavam essa droga,

Eles tinham clareza que haviam produzido um inibidor de recaptação de serotonina, mas tinham menos clareza no início sobre para que esse composto poderia ser usado. Após os primeiros estudos de toxicidade em animais terem demonstrado que o composto era provavelmente seguro em humanos, a Lilly convocou uma série de encontros com investigadores clínicos para testar e determinar qual mercado poderia existir para um inibidor de recaptação de serotonina. [...] Foi apenas no início da década de 1980, [...] com a percepção de que o tamanho do mercado de antidepressivos começou a mudar, que a Lilly acelerou o desenvolvimento da fluoxetina como um antidepressivo. O estudo clínico que evidenciou suas propriedades antidepressivas tornou-se disponível apenas em 1985 (Healy 1997:167-168).

No livro de Goodman e Gilman, a partir da $3^{\mathrm{a}}$ edição, há um capítulo que trata dos "autacoides", ${ }^{26}$ incluindo substâncias atuantes em várias partes 
do organismo, mas, na ocasião, sem suas funções identificadas. Entre as principais substâncias estavam a histamina e a serotonina. Presentes em todo o organismo (nas células do trato gastrointestinal, nas plaquetas e nas sinapses neuronais), prometiam um futuro promissor àqueles que trabalhavam com o desenvolvimento de medicamentos, mesmo que ainda não se soubesse para quais situações clínicas (Goodman \& Gilman 1965:614-615). Em determinado momento, percebeu-se que medicamentos atuantes sobre a serotonina poderiam ser úteis nos quadros com sintomas depressivos. Atualmente, os ISRS são as drogas que expandiram os domínios dos antidepressivos para várias situações de saúde além do tratamento de situações relacionadas ao humor.

Se os tricíclicos já eram utilizados para outras situações (enurese noturna, dores crônicas, entre outros), os ISRS ganham espaço de forma muito mais ampliada, em grande parte por seus menores efeitos colaterais:

Em adição ao seu uso na síndrome de depressão maior no adulto, os vários agentes antidepressivos têm encontrado ampla utilidade em várias outras desordens psiquiátricas que podem ou não estar psicobiologicamente relacionadas às desordens do humor. O estímulo para encontrar novas indicações [desses medicamentos] tem aumentado com o advento dos agentes mais novos, que são menos tóxicos, simples de usar e muitas vezes mais aceitos tanto por médicos como por pacientes (Baldessarini 1996:445; 2001:468, grifo nosso).$^{27}$

Eles deixam de ser apenas "antidepressivos", embora mantenham este nome, para se tornarem drogas de múltiplos usos, impulsionadas por seus efeitos "menos tóxicos" e por sua "simplicidade de uso". O destaque vai para a parte grifada, em que se abrem novas possibilidades de uso para essas drogas, que passam a ter outras indicações além de ações "antidepressivas", tais como transtorno de ansiedade, fibromialgia, anorexia nervosa, bulimia, diabetes e outras síndromes neuropáticas, entre outros (Baldessarini 1996:446).

\section{Considerações finais}

Se "depressão" foi um termo utilizado no sentido de "baixo funcionamento" ou "redução da função" no início de sua introdução na biomedicina, por volta de 1860 (Berrios 1996), os medicamentos para tratar situações clínicas relacionadas aos sintomas depressivos aparecem somente em torno de 1950. Anteriormente, alguns medicamentos eram empregados para tratar esses 
sintomas, mas não como uma classe de drogas específicas. No livro-texto de farmacologia de Goodman e Gilman, essas drogas aparecem de forma explícita a partir da $3^{\mathrm{a}}$ edição (1965), ainda que introduzidas na prática biomédica anos antes. Esta é uma característica citada pelos autores do livro no prefácio de suas diferentes edições, lembrando que o conhecimento científico está em constante mudança, e o tempo necessário para a pesquisa daquilo que vai ser inserido na obra reflete os acontecimentos ocorridos alguns anos antes.

A partir da $4^{\mathrm{a}}$ edição (1965), aparece o termo "antidepressivo" para caracterizar um conjunto de drogas utilizado para tratar sintomas e síndromes relacionadas à depressão, situação clínica que passa a ser categorizada como entidade nosológica na segunda metade do século XX. Esse período, de mudanças significativas em todo o mundo, se reflete na biomedicina e na produção de medicamentos. Uma das grandes características dessa época foi a expansão industrial, e "as principais inovações que começaram a transformar o mundo assim que a guerra acabou talvez tenham sido as do setor químico e farmacêutico" (Hobsbawm 1995:265). Vale lembrar que a indústria, além de oferecer um produto a ser usado no tratamento de problemas de saúde, visa ao lucro, e este aspecto não pode ser dissociado do desenvolvimento dos medicamentos: além de úteis, eles devem proporcionar ganhos financeiros.

A partir da análise do livro-texto, é possível perceber que, embora a farmacologia possua certa autonomia (tanto em seus métodos quanto nas suas regras de desenvolvimento), está em constante diálogo com a prática clínica de cada momento histórico. Apesar de hipóteses teóricas e pesquisas com drogas influenciarem o desenvolvimento de novos fármacos, quem determina quais são as situações de sofrimento que merecem ser tratadas ou não com medicamentos ainda é a prática clínica, e isto aparece nas diferentes edições do livro-texto. Como afirma Canguilhem (2006 [1966]), "são os insucessos da vida [o que consideramos doença ou 'mau funcionamento'] que chamam e que sempre chamaram - a atenção" (:170). São os critérios clínicos que impulsionam o desenvolvimento da farmacologia, e não o contrário.

O quanto a indústria farmacêutica influencia a criação ou a expansão dos diagnósticos clínicos para promover a expansão do mercado de medicamentos não foi diretamente observado no livro-texto, mas esta é uma questão que tem sido levantada por diversos autores. Situações de vida em que aparecem sintomas como a tristeza, a ansiedade ou o medo têm sido apontadas como depressão, síndrome do pânico, fobia social, entre outras condições hoje categorizadas como "doença" (Healy 1997; Pignarre 2001; Conrad 2007; Sismondo 2007). O uso de medicamentos antidepressivos para 
tratar esses sintomas tem sido uma das ferramentas para corroborar essas situações como "problemas médicos", e a ampliação do espaço que essas substâncias ocupam no livro de farmacologia reflete a extensão de seu uso na prática médica contemporânea.

Foi possível observar no decorrer da análise do livro-texto que não são as teorias farmacológicas, que tentam explicar funcionamentos fisiopatológicos, que determinam o uso das drogas. Mesmo quando são utilizados argumentos a partir dos estudos dessa área do conhecimento, há sempre a ressalva dos autores de que ainda não se comprovou esta ou aquela teoria, que há "indícios" de que esta ou aquela substância atua em determinada via fisiopatológica, mas não está estabelecida uma relação causal a partir do conhecimento farmacológico. Também é possível perceber que seu desenvolvimento acompanhou o interesse dos pesquisadores em "descobrir" funções para substâncias endógenas, tais como a histamina e a serotonina. A partir do momento em que se percebeu que elas estavam amplamente distribuídas no organismo e participavam de várias funções, essas substâncias passaram a ser exaustivamente estudadas, como descrevem Goodman e Gilman (1964 [1955]).

Ao mesmo tempo em que isso acontecia no âmbito da farmacologia, na prática clínica percebeu-se que os problemas relacionados à saúde mental ocupavam grande espaço na prática médica, ao aparecerem cada vez mais indivíduos com sofrimento moderado, em nível ambulatorial, além daqueles internados. O tratamento medicamentoso surge como estratégia para lidar com um número grande de pessoas então diagnosticadas com esses problemas. Novamente, um estímulo externo à farmacologia.

Nas últimas edições do livro-texto, também foi possível perceber a ampliação do uso dos medicamentos que agem sobre a serotonina para além dos quadros relacionados a sintomas depressivos. Em um futuro próximo, a denominação desses medicamentos poderá deixar de ser "antidepressivos", pois seu uso em diferentes situações clínicas já ocorre, como no caso dos "distúrbios de ansiedade" ou de "outros sintomas que podem, ou não, ter relação com as desordens do humor" (Baldessarini 1996:446). Uma das razões para esse uso ampliado, apontada no próprio texto de farmacologia, é que medicamentos da classe dos ISRS têm menos efeitos colaterais do que outros antidepressivos usados para os mesmos problemas. No entanto, sabemos por experiência anterior que muitos medicamentos considerados seguros em determinadas épocas (como os barbitúricos e BZD) só tiveram seus efeitos colaterais importantes "descobertos" no decorrer de vários anos de uso.

A abordagem do contexto histórico do desenvolvimento de medicamentos e do contexto sociocultural de seu uso pode contribuir para compreender 
melhor sua utilização e, com isso, instrumentalizar os profissionais que lidam com a dor e o sofrimento humanos na sua prática diária. Mesmo que em alguns momentos a intervenção médica ocorra com o emprego de um medicamento, resulta necessário reconhecer os limites e as dificuldades desse tipo de intervenção, de modo tal que não se reduza a prática terapêutica a uma mera busca de alteração de neurotransmissores ou substâncias endógenas.

Os autores do livro-texto de farmacologia sugerem, em suas primeiras edições, que o doente é o foco do encontro terapêutico, que o médico deve ouvi-lo e considerar o contexto em que o paciente está inserido para auxiliá-lo a lidar com a situação de sofrimento, algo que no decorrer dos anos vai desaparecendo do discurso. O resgate deste e de outros âmbitos (como os aspectos históricos) em um livro de farmacologia, relativizando o papel dessa ciência e contextualizando o desenvolvimento dos fármacos, como acontecia nas publicações iniciais dessa obra, certamente pode contribuir para uma abordagem médica não reducionista.

Recebido em 02 de janeiro de 2013

Aprovado em 23 de julho de 2013

Silvia Cardoso Bittencourt é Professora do Curso de Medicina da Universidade do Sul de Santa Catarina (UNISUL), Unidade Pedra Branca. E-mail: <scbflor@ hotmail.com>

Sandra Caponi é professora do Departamento de Sociologia e Ciências Políticas da Universidade Federal de Santa Catarina. E-mail: <sandracaponi@gmail.com>

Sônia Maluf é professora associada III da Universidade Federal de Santa Catarina, docente do PPGAS/UFSC e do PPGICH/UFSC. E-mail: <maluf@cfh. ufsc.br>

\section{Notas}

${ }^{1}$ Substâncias psicoativas são aquelas capazes de mudar a consciência, o humor e os pensamentos por meio da ação em receptores cerebrais específicos (WHO 2004). Medicamento psicoativo é uma substância psicoativa utilizada para tratar problemas de saúde. 
${ }^{2}$ Psicotrópicos: substâncias que atuam no SNC sedando, estimulando ou alterando o humor. Podem ser didaticamente divididos em: a) ansiolíticos-sedativos, b) antidepressivos, c) estabilizantes do humor, d) neurolépticos ou antipsicóticos (Baldessarini 2001:447).

${ }^{3}$ Psicofarmacologia: subespecialidade desenvolvida a partir da década de 1950. Lida com o uso dos medicamentos psicoativos utilizados para tratar problemas de saúde. Estuda a química, as ações e a farmacologia clínica das drogas que atuam no SNC (Baldessarini 2001:447).

${ }^{4}$ Neurolépticos: termo para designar as drogas antipsicóticas. Owens (1996) afirma que o uso deste termo, mais comum que "antipsicótico", ocorre porque enfatiza as ações neurológicas dessas drogas.

${ }^{5}$ Inibidores da MAO: Inibidores da Enzima Monoaminoxidase que fazem a recaptação de neurotransmissores, como as catecolaminas e a serotonina na fenda sináptica (Brunton, Lazo \& Parker 2006).

${ }^{6}$ Iproniazida; descoberta a partir da hidrazina, substância carburante utilizada em foguetes durante a Segunda Guerra. Quando a guerra terminou, grandes indústrias químicas recuperaram os estoques desta substância e testaram seus derivados para diferentes doenças (Dagognet \& Pignarre 2005:33).

${ }^{7}$ Fenotiazinas: substâncias com ação anti-histamínica, utilizadas inicialmente para tratar quadros alérgicos e que, entre outros efeitos, atuam no sistema Nervoso Central. A clorpromazina é uma fenotiazina e foi a primeira droga a ser utilizada para tratar problemas psiquiátricos (Dagognet \& Pignarre 2005; Fangmann 2008).

${ }^{8}$ A empresa de Johann Rudolf Geigy-Gemuseus foi fundada na Suíça em 1758, comercializando corantes e medicamentos. Em 1914, torna-se uma empresa de capital aberto e passa a se chamar J.R. Geigy Ltda. Em 1970, esta empresa se uniu à Ciba, que possuía um departamento farmacêutico desde 1938, formando a Ciba-Geigy Ltda. Em 1992, a Ciba, nome adotado na fusão de 1970, se une à Sandoz (empresa suíça fundada em 1886), formando a Novartis. A partir de 1996, são incorporadas à Novartis outras empresas de medicamentos e vacinas, tornando-a uma das maiores indústrias deste segmento na atualidade (Novartis 2012; Sandoz 2009).

${ }^{9}$ Antipiréticos (ou antitérmicos) são aqueles medicamentos capazes de reduzir a temperatura corporal, utilizados para baixar a febre (Schenkel, Menguel \& Petrovick 2004:119).

${ }^{10} \mathrm{O}$ elemento brometo foi descoberto em 1826. Nove anos depois, seu sal de potássio foi introduzido na Farmacopeia Inglesa e, em 1850, foi observada sua ação no SNC. Goodman e Gilman referem que, na época da edição do livro, em 1941, não se sabia qual sua forma de ação, mas já se sabia que o íon brometo por si só tinha ação sedativa (Goodman \& Gilman 1947 [1941]:155). 
${ }^{11}$ Halogênio: utilizado como sinônimo de brometo.

${ }^{12}$ Fadiga de combate: desordem relacionada à ansiedade (sintomas de estresse relacionados ao pós-guerra). Hoje não é mais conhecida com esta denominação. Um equivalente atual seria o quadro de ansiedade pós-traumática. Disponível em: <http://www.britannica.com/EBchecked/topic/127295/combat-fatigue> . Acesso em: 15/04/2010.

${ }^{13}$ Este excerto é parte do livro Goodman \& Gilman (1965) e foi escrito por Jarvik.

${ }^{14}$ Este excerto faz parte do livro Goodman \& Gilman $(1975,5$. ed.) e foi escrito por Byck (1975).

15 O Diagnostic and Statistical Manual of Mental Disorders II (DSM II), da Americam Psychiatric Association, foi editado em 1968.

${ }^{16}$ Baldessarini é autor de capítulos do livro-texto de Goodman e Gilman nas $6^{\mathrm{a}}, 7^{\mathrm{a}}, 8^{\mathrm{a}}, 9^{\mathrm{a}}, 10^{\mathrm{a}}$ e $11^{\mathrm{a}}$ edições.

${ }^{17}$ Librium ${ }^{\circledR}$ e Valium ${ }^{\circledR}$ são os nomes comerciais de dois compostos benzodiazepínicos amplamente utilizados e reconhecidos pelo nome comercial nas últimas décadas do século XX.

${ }^{18}$ Timolépticos: drogas que alteram o humor, como os tricíclicos, inibidores da MAO e sais de lítio. Disponível em: < http://medical-dictionary.thefreedictionary. com/thymoleptic $>$. Acesso em: 22/04/2010.

${ }^{19}$ Compostos dibenzazepínicos são os compostos tricíclicos, como a imipramina e a amitriptilina.

${ }^{20}$ 5-HT: 5-hidróxi-triptamina, sinônimo de serotonina.

${ }^{21}$ A parte em itálico é idêntica à que aparece na $10^{\mathrm{a}}$ edição (2001), e a parte inicial desta citação é quase idêntica (Baldessarini 2001:451).

${ }^{22}$ Desde 1999, a Astra $A B$, uma empresa sueca, associou-se ao grupo inglês Zeneca, formando a AstraZeneca Internacional. Disponível em: < http://www.astrazeneca.com/about-us/history/>. Acesso em: 22/04/2010.

${ }^{23}$ Lundbeck: empresa farmacêutica que trabalha com medicamentos psicoativos (Lundbeck 2000).

${ }^{24}$ Eli Lilly: empresa de medicamentos fundada em 1876. Para mais detalhes ver: < http://www.lilly.com/about/>. Acesso em: 25/04/2010.

${ }^{25}$ 1986, segundo o site da própria Eli Lilly (Disponível em: < http://www.prozac. com/Pages/index.aspx>. Acesso em: 30/04/2010). 
${ }^{26}$ Autacoide: substância fisiologicamente ativa, como a bradicinina ou angiotensina, produzida e ativa no interior do organismo (Merrian-Webster 2012). Segundo Goodman e Gilman (1965), na $3^{a}$ edição do livro, estavam incluídas nesta categoria também a serotonina e a histamina, pois não se conhecia na época o papel dessas substâncias. Eles afirmam: "Qual o significado [importância] desse grupo de autacoides? Qual o seu papel no corpo [body]? Qual o seu valor como medicamentos [drugs] e qual o seu lugar na terapêutica? Infelizmente, somente poucas respostas imprecisas podem ser dadas para estas questões. O fato certo pelo qual essas substâncias foram classificadas sob o título impreciso de "autacoides" é, em certo sentido, uma confissão de que, no presente, a evidência não permite uma classificação funcional tal como, por exemplo, a de hormônio ou neuro-humor" (Goodman \& Gilman 1965:615).

${ }^{27}$ Este excerto aparece também na $10^{\mathrm{a}}$ edição do livro (Hardman, Limbird \& Gilman 2001:468) e na $11^{a}$ edição (Brunton, Lazo \& Parker 2006:449).

\section{Referências bibliográficas}

AGUIAR, Adriano Amaral de. 2004. A psiquiatria no divã. Entre as ciências da vida e a medicalização da existência. Rio de Janeiro: Relume-Dumará.

AUCHEWSKI, Luciana et alli. 2004. "Avaliação da orientação médica sobre os efeitos colaterais de benzodiazepínicos". Revista Brasileira de Psiquiatria, 26(1):24-31.

BALDESSARINI, Ross J. 2006. “Drug therapy of depression and anxiety disorders". In: L.L. Brunton; J.S. Lazo \& K.L. Parker (edit. assoc.), Goodman and Gilman's the pharmacological basis of therapeutics. 11. ed. USA: McGraw-Hill Companies. pp. 429-460.

. 2001. "Drugs and the treatment of psychiatric disorders: depression and anxiety disorders". In: J.G. Hardman; L.E. Limbird \& A.G. Gilman (edit. assoc.), Goodman and Gilman's the pharmacological basis of therapeutics. 10. ed. USA: McGraw-Hill Companies. pp. 447-484.
— 1996. "Drugs and the treatment of psychiatric disorders: depression and anxiety disorders". In: J.G. Hardman \& L.E. Limbird (edit. assoc.), Goodman and Gilman's the pharmacological basis of therapeutics. 9. ed. International edition: McGraw-Hill Companies. pp. 431-460.

. 1990. "Drugs and the treatment of psychiatric disorders". In: A.G. Gilman \& T.W. Rall (edit. assoc.), Goodman and Gilman's the pharmacological basis of therapeutics. 8. ed. New York: Pergamon Press. pp. 383-435. . 1985. "Drugs and the treatment of psychiatric disorders". In: A.G. Gilman \& L.S. Goodman (edit. assoc.), Goodman and Gilman's the pharmacological basis of therapeutics. 7. ed. New York: Pergamon Press. pp. 387-445.

.1983. "Medicamentos e o tratamento de doenças psiquiátricas". In: A.G. Gilman \& L.S. Goodman (edit. assoc.), Goodman e Gilman - as bases 
farmacológicas da terapêutica. 6. ed. Rio de Janeiro: Guanabara Koogan. pp. 347-394.

BERRIOS, German E. 1996. "Affect and its disorders". In: The history of mental symptoms. Descriptive psychopathology since the nineteenth century. Cambridge, UK: Cambridge University Press. pp. 289-331.

BRUNTON, L. L.; LAZO, J. S. \& PARKER, K. L. (edit. assoc.). 2006. Goodman and Gilman's the pharmacological basis of therapeutics. 11. ed. USA: McGrawHill Companies.

BURKE, Peter. 2002. História e teoria social. São Paulo: Unesp.

BYCK, Robert. 1975. "Drugs and the treatment of psychiatric disorders". In: L.S. Goodman \& A. Gilman (edit. assoc.), The pharmacological basis of therapeutics. New York: Mcmillan. pp. 152-200.

CANGUILHEM, Georges. 2006 [1966]. O normal e o patológico. Rio de Janeiro: Forense Universitária.

CONRAD, Peter. 2007. The medicalization of society. On transformation of human conditions into treatable disorders. Baltimore: John Hopkins University Press.

DAGOGNET, François \& PIGNARRE, Philippe. 2005. 100 mots pour comprendre les medicaments. Comment on vous soigne. Paris: Les Empêcheurs de Penser en Rond.

FANGMANN, Peter et alli. 2008. "Half a century of antidepressant drugs: on the clinical introduction of monoamine oxidase inhibitors, tricyclics and tetracyclics. Part II: tricyclics and tetracyclics". Journal of Clinical Psychopharmacology, 28(1):1-4.

GILMAN, Alfred Goodman et alli (edit.). 1990. Goodman e Gilman. As bases farmacológicas da terapêutica. $8^{\mathrm{a}} \mathrm{ed}$. Rio de Janeiro: Guanabara Koogan. - 1985. Goodman and Gilman's: the pharmacological basis of therapeu- tics. 7. ed. New York: Macmillan Publishing Company.

GILMAN, Alfred Goodman; GOODMAN, Louis S. \& GILMAN, Alfred (edits.). 1983 [1980]. As bases farmacológicas da terapêutica. $6^{\text {a }}$ ed. Rio de Janeiro: Guanabara Koogan.

GOODMAN, Louis S. \& GILMAN, Alfred. 1975. The pharmacological basis of therapeutics. 5. ed. New York: Macmillan Publishing Co.

- 1970. The pharmacological basis of therapeutics. 4. ed. New York: Macmillan Publishing Co.

- 1965. The pharmacological basis of therapeutics. A textbook of pharmacology, toxicology, and therapeutics for physicians and medical students. 3. ed. New York: Mc Millan Company.

_. 1964 [1955]. The pharmacological basis of therapeutics. A textbook of pharmacology, toxicology and therapeutics for physicians and medical students. 2. ed. New York: Macmillan Company.

- 1947 [1941]. The pharmacological basis of therapeutic. A textbook of pharmacology, toxicology and therapeutics for physicians and medical students. 1. ed. New York: Macmillan Co.

HARDMAN, Joel G.; LIMBIRD, Lee E. \& GILMAN, Alfred Goodman (edits. assocs.). 2001. Goodman and Gilman's the pharmacological basis of therapeutics. 10. ed. USA: McGraw-Hill Companies.

HARDMAN, Joel G. et alli. 1996. Goodman and Gilman's the pharmacological basis of therapeutics. 9. ed. New York: McGraw-Hill.

HEALY, David. 1998. "Pionners in psychopharmacology". International Journal of Neuropsychopharmacology, 1(2):191-194. . 1997. The antidepressant era. Cambridge, MA: Harvard University Press. 
HOBSBAWM, Eric. 1995. A era dos extremos. O breve século XX: 1914-1991. São Paulo: Companhia das Letras.

HORWITZ, Allan V. \& WAKEFIELD, Jerome C. 2007. The loss of sadness. How psychiatry transformed normal sorrow into depressive disorder. New York: Oxford University Press.

JARVIK, Murray E. 1970. “Drugs used in the treatment of psychiatric disorders". In: A. Goodman \& L.S. Gilman (eds.), The pharmacological basis of therapeutics. A textbook of pharmacology, toxicology, and therapeutics for physicians and medical students. 4. ed. New York: Mc Millan Company. pp. 151-203.

. 1965. "Drugs used in the treatment of psychiatric disorders". In: A. Goodman \& L.S. Gilman (eds.), The pharmacological basis of therapeutics. A textbook of pharmacology, toxicology, and therapeutics for physicians and medical students. 3. ed. New York: Mc Millan Company. pp. 159-214.

LACEY, Hugh. 1998. Valores e atividade científica. São Paulo: Discurso Editorial.

LEITE, Raquel Crosara Maia. 2004. A produção coletiva do conhecimento científico: um exemplo no ensino de genética. Tese de Doutorado, Programa de Pós-Graduação em Educação, Universidade Federal de Santa Catarina.

LEWONTIN, R. 1993 [1991]. Biology as ideology: the doctrine of DNA. Ontario: Harper Perennial.

LOPEZ-MUNOZ, Francisco et alli. 2004. "Historical approach to reserpine discovery and its introduction in psychiatry". Actas Espanõlas de Psiquiatría, 32(6):387-395.

LUNDBECK. 2000. Lundbeck Annual Report. Disponível em: <http://www. materials. lundbeck.com/lundbeck/82/104 > . Acesso em: 25/04/2010.
MAZANA, J.S.; PEREIRA, J. \& CABRERA, R. 2002. "Cincuenta años de clorpromazina". Revista Espanõla de Sanidad Penitenciaria, 4:101-113.

MERRIAN-WEBSTER. 2012. "Autacoid". Disponível em: <http://www.merriam-webster.com/medical/autacoid > Acesso em: 28/09/2012.

MOMMSEN, Wolfgang J. 1982. "La historia". In: L. Apostel et alli (eds.), Interdisciplinariedad y ciencias humanas. Madrid/ Paris: Tecnos/ Unesco. pp. 236-251.

NASTASY, H.; RIBEIRO M.; MARQUES, A.C.P.R. 2008. "Abuso e dependência dos benzodiazepínicos". In: Projeto Diretrizes. IAMB/CFM. Disponível em: $<$ http://www. projetodiretrizes.org. br/projeto_diretrizes/004.pdf > . Acesso em: 23/04/2010.

NOGUEIRA, Conceição. 2008. "Análise(s) do discurso: diferentes concepções na prática de pesquisa em psicologia social". Psicologia: Teoria e Pesquisa, 24(2):235-242.

. 2001. "A análise do discurso". In: L. Almeida \& E. Fernandes (eds.), Métodos e técnicas de avaliação: novos contributos para a prática e investigação. Braga: CEEP. pp. 15-48.

NOVARTIS. 2012. "Geigy, Ciba e Sandoz (1758-1970)". Disponível em: < http:// www.novartis.com.br/_sobre_novartis/ historia/geigy_ciba_sandoz.shtml>. Acesso em: 20/09/2010.

ORLANDI, Eni. 2005. "Scientific discourse and interpretation". Multiciência, 4. Disponível em: <http://www.multiciencia.unicamp.br/artigos_04/a_02. pdfhttp://www. multiciencia.unicamp. br/artigos_04/a_02_.pdf $>$. Acesso em 12/10/2009. . 1999. Análise do discurso. Princípios e procedimentos. Campinas: Pontes.

OWENS, D.G.C. 1996. "Advances in psychopharmacology-schizophrenia". British Medical Bulletin, 52(2):556-74. 
PIGNARRE, Philippe. 2001. Comment la depression est devenue une epidemie. Paris: La Découverte \& Syros. . 1999. O que é o medicamento? Um objeto estranho entre ciência, mercado e sociedade. São Paulo: 34.

SANDOZ. 2009. "História". Disponível em: <http://www.sandoz.com.br/site/ br/ sobre_a_sandoz/historia/index. shtml>. Acesso em: 20/02/2010.

SCHENKEL, Eloir Paulo; MENGUE, Sotero Serrante \& PETROVICK, Pedro Ros (orgs.). 2004. Cuidado com os medicamentos. Florianópolis/Porto Alegre: UFSC/ UFRGS.

SISMONDO, Sergio. 2007. "Ghost management: how much of the medical literature is shaped behind the scenes by the pharmaceutical industry?" Plos Medicine, 4(9):1429-1433.

TURNQUIST, Kevin. 2002. "Are we becaming a nation of depressives?". The Humanist, 62(5):27-33.

VAN DIJK, Teun A. 1999. "El análisis crítico del discurso". Anthropos, 186:23-36.

WHO (WORLD HEALTH ORGANIZATION). 2004. Neuroscience of psychoactive substance use and dependence. Disponível em: <http://www.who. int/substance_abuse/publications/en/ Neuroscience_E.pdf $>$. Acesso em: 14/07/2008.

. 1983. "Use and abuse of benzodiazepines". Bulletin of the World Health Organization, 61(4):551-562.

WOODS, James H.; KATZ, Jonathan L. \& WINGER, Gail. 1988. "Use and abuse of benzodiazepines". JAMA, 260(23):3476-3480. 


\section{Resumo}

Este estudo investiga como os antidepressivos foram apresentados a estudantes e profissionais da área médica a partir da análise de um livro-texto de farmacologia com projeção mundial. Através de uma perspectiva histórica e etnográfica, foram analisadas onze edições da obra (1941-2006), com auxílio de recursos da Análise de Discurso. Esses medicamentos aparecem no livro-texto a partir da $3^{a}$ edição (1965). Nas edições do século XX, há referência ao uso ampliado de medicamentos psicoativos e o alerta para que não se faça uso inadequado dessas drogas, lembrando a importância da contextualização clínica do paciente a ser medicado. Nas edições do século XXI, há menos referências a essa contextualização e aos aspectos históricos do desenvolvimento dos medicamentos. Este estudo reafirma a importância da abordagem de aspectos históricos e socioculturais do desenvolvimento da farmacologia e de fármacos, como os antidepressivos, bem como da relação desses aspectos com a prática clínica e a divulgação científica dos medicamentos.

Palavras-chave Uso de antidepressivos, Drogas psicoativas, Pesquisa interdisciplinar, História da farmacologia, Indústria farmacêutica.

\section{Abstract}

Based on the analysis of a pharmacological textbook with a global projection, this study investigates how antidepressants were presented to students and medical professionals. From a historical and ethnographic perspective, eleven editions of the work (1941-2006) were analyzed with aid from the resources of the Analysis Discourse. These drugs make their first appearance in the 3rd edition (1965) of the textbook. In the twentieth century editions, there is reference to the expanded use of psychoactive medications and a warning concerning the inappropriate use of these drugs, which notes the importance of the clinical context of the patient to be medicated. In the editions of the twenty-first century, there is less reference to that contextualization and the historical aspects of the drug development. This study restates the importance of addressing historical and sociocultural aspects of pharmacological development and drugs such as antidepressants, as well as the relationship between these aspects of clinical practice and the scientific dissemination of the drugs.

Key words Drug use, Interdisciplinary research, Pharmacological history, Pharmaceutical industry, Psychoactive drugs. 